\title{
A Polymeric $\mathrm{FeCl}_{3}$ Catalyst for Deprotection of Acetonides
}
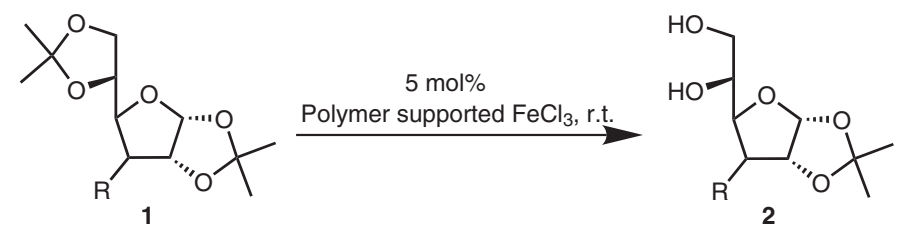

$87-94 \%$ yield

Significance: Highly chemoselective deprotection of diacetone sugars bearing various functional groups was achieved at room temperature for 30 minutes using poly(4-vinylpyridine)-supported ferric chloride (5 mol\%) to give the corresponding terminal diols 2 in $87-94 \%$ yields (15 substrates). This method was found to be of good functional compatibility with acid-sensitive groups such as TBDMS, THP, OBn, OBz, and OAc. The polymeric catalyst was readily recycled without lenching of ferric chloride.
Comment: Several examples of protection/deprotection of 1,2-diols with acetonide using $\mathrm{FeCl}_{3}$ (e.g., A. Fadel, R. Yefsah, J. Salaün Synthesis 1987, 37-39) have been reported so far. 